\title{
Presence of Cardiovascular Risk as Diabetes Mellitus Progresses
}

\author{
Chundusu $\mathrm{CM}^{*}$ \\ Department of Medicine, College of Medical Sciences, University of Jos, Nigeria
}

*Corresponding author: Chundusu CM, Department of Medicine, College of Medical Sciences, University of Jos, PMB 2084, Jos, Plateau State, Nigeria, Tel: 08037032797, E-mail: calebchundusu@yahoo.com

Citation: Chundusu CM (2018) Presence of Cardiovascular Risk as Diabetes Mellitus Progresses. J Card Disord Therapy 1: 105

Article history: Received: 05 March 2018, Accepted: 04 April 2018, Published: 09 April 2018

\begin{abstract}
Background: As individual lives with Diabetes Mellitus, the emergence and progression cardiovascular risks are relevant in the management of patients. While some appear early and last long some appear late and cause patients condition to deteriorate rapidly.

Objectives: To assess the relative progression of various cardiovascular risk factors considering the duration of diabetes mellitus.

Method: One hundred and ninety asymptomatic people living with diabetes mellitus were evaluated considering time as at first diagnosis of DM irrespective of the initial care given. Cardiovascular risk factors were compared in 6 sub-grouping based on duration of diabetes mellitus.
\end{abstract}

Results: Increasing duration of DM showed significant increase with left ventricular mass, uric acid and creatinine.

Conclusion: cardiovascular risk no doubt increases as the duration of DM increases and more so on the heart and the kidney.

Keywords: Diabetes duration; First specialist clinic visit; Cardiovascular risk

\section{Introduction}

Diabetes Mellitus (DM) is a common chronic endocrine disorder of insulin secretion or function resulting in the disturbances of carbohydrate, fats and protein metabolism characterised by hyperglycemia. Cardiovascular complications are the most important cause of morbidity and mortality. Epidemiological and clinical trial data have estimated that $60-70 \%$ of all patients with type 2 diabetes will die from CVD [1,2]. DM adds about 15 years in terms of cardiovascular risk compared to normal individuals [3].

Duration of DM is a very important factor in the development of cardiovascular complications irrespective of the quality of care given [4,5]. Overt nephropathy caused by glomerulosclerosis appears 10-15years after the onset of insulin dependent DM (IDDM) and after 5-10years in patients with non-insulin dependent DM (NIDDM) [5,6]. Although duration of DM was based on subject's recall, using this might give an idea of the possible progression of complications present. Most patients in our environment present to the specialist clinic only after having consulted several other treatment modalities ranging from self-medications, faith based interventions, nearby chemists or laboratories.

This work compares the association of progression duration of diabetes mellitus with some common cardiovascular risk identifiers.

\section{Methods}

This was an observation made from a carotid intima-media thickness (original study) in study between 2011 and 2012 . A cross sectional study among adults 18 years old or above. Subjects were consecutively recruited while being evaluated on first specialist clinic visit at the medical out-patient clinic at the Jos University Teaching Hospital. They are not known to be on be managed of any complication, and all the patients must have given an informed consent. The subjects less than eighteen years, regular clinic patients and those that declined participating are excluded from the study. The original study was duly approved by the Ethics Committee of the Jos University Teaching Hospital, Jos. Nigeria.

A questionnaire was used to obtain bio-data and relevant clinical history with durations of illness. A general clinical examination was carried out on the subjects. Fasting plasma glucose, waist circumference, BMI were measured and Blood pressure. 


\section{Laboratory analysis}

Seven (7) mls of fasting venous blood sample was taken for serum createnin, lipids and uric acid level.

\section{Ultrasonography}

The carotid intima-media thickness of the far wall of proximal $10 \mathrm{~mm}$ of the two common carotid arteries were determined using the $8.0 \mathrm{MHz}$ probe of real time B mode ultrasound imager (ALOKA SSD 4000 ultrasound system Japan).

A 3.5 MHz probe was then used to obtain 2D guide M-mode left ventricular measurements. The Left ventricular mass was obtained using the Devereux formula software. These factors measured were based on the original study and only analysed because they were available.

\section{Statistical analysis}

Subjects were grouped based on the duration of DM into less than 1 year, 1-5 years, 6-10 years, 11-16 years, 16-20 years and greater than 20 years. Continuous variables were expressed as means. Analysis was done with both Epi-info 3.5.1 and ANOVA test was done to compare means of the six groups. Statistical significant variation was $\mathrm{p}<0.05$

\section{Results}

A total data of 190 participants were analysed. Subjects were between the ages of 21 and 88 years. The total mean age was $55.29+$ 14 years.

The subjects were mostly already on anti-diabetic agent/drug Metformin (70\%), sulfonylurea Glibenclamide (52\%), implying that most subjects were type 2 diabetics. The thiaozolidinediones and glucosidase inhibitors and other relatively newer anti-diabetic drugs were prescribed to very few subjects. Hypertension was the most common co-morbidity (51\%), and the most common anti-hypertensive drugs taken by diabetic-hypertensive were of the angiotensin receptor blockers (ARB) or angiotensin converting enzyme inhibitor(ACEI) (50\%) group followed by Calcium channel blockers (30\%). Diuretics were noted in $25 \%$ of subjects.

The characteristics of the subject groups and the risk factors are represented by the table below

\begin{tabular}{|c|c|c|c|c|c|c|c|}
\hline Duration of DM & $<\mathbf{l y r}$ & $\mathbf{1 - 5 y r s}$ & $\mathbf{6 - 1 0 y r s}$ & $\mathbf{1 1 - 1 5 y r s}$ & $\mathbf{1 6 - 2 0 y r s}$ & $>\mathbf{2 0 y r s}$ & p-value \\
\hline $\mathbf{N}(\%)$ & $34(18 \%)$ & $76(40 \%)$ & $44(23 \%)$ & $22(11 \%)$ & $10(5 \%)$ & $4(2 \%)$ & \\
\hline Male (\%) & $18(53 \%)$ & $28(37 \%)$ & $12(27 \%)$ & $8(36 \%)$ & $6(60 \%)$ & $2(50 \%)$ & \\
\hline Age (yrs) & 50.8 & 52.3 & 62.0 & 60.8 & 66.8 & 63 & \\
\hline WC (cm) & 100.1 & 95.5 & 100.3 & 102.6 & 97.5 & 90.0 & 0.43 \\
\hline SBP (mmHg) & 124.4 & 130.5 & 133.0 & 141.6 & 145.0 & 160.0 & 0.07 \\
\hline DBP (mmHg) & 81.8 & 83.2 & 78.4 & 83.4 & 79.0 & 85.0 & 0.73 \\
\hline LVM (g) & 272.1 & 230.9 & 255.2 & 282.6 & 392.8 & 219.0 & $0.01^{*}$ \\
\hline CIMT (mm) & 0.7565 & 0.7558 & 0.8545 & 0.8291 & 0.8180 & 0.8550 & 0.10 \\
\hline UA(mmol/dl) & 333.3 & 342.1 & 382.0 & 419.6 & 417.7 & 490.0 & $0.03^{*}$ \\
\hline Chol T(mmol/dl) & 4.46 & 4.79 & 4.94 & 5.67 & 4.65 & 6.70 & 0.14 \\
\hline HDL(mmol/dl) & 1.18 & 1.17 & 1.30 & 1.25 & 1.23 & 1.0 & 0.87 \\
\hline TG (mmol/dl) & 1.41 & 1.46 & 0.92 & 1.26 & 1.71 & 1.80 & 0.36 \\
\hline FBS (mmol/dl) & 9.9 & 9.6 & 10.5 & 9.1 & 9.4 & 8.6 & 0.70 \\
\hline Cr (mmol/dl) & 91.76 & 87.79 & 96.00 & 99.65 & 91.0 & 257.20 & $0.00^{*}$ \\
\hline
\end{tabular}

Keys: WC: waist circumference; SBP: systolic blood pressure; DBP: diastolic blood pressure; LVM: left ventricular mass; CIMT: carotid intima-media thickness, UA: uric acid, chol T- total cholesterol; HDL: high dense lipoprotein; FBS: fasting blood sugar; Cr: createnin; P: value was significant $\left(^{*}\right)$

Table 1: Risk Factors in Different Groupings of Duration of DM

The duration of DM ranged from lyear to 30 years, with a mean of $6.99+5.97$ years and median of $5 y e a r s$. The $25^{\text {th }}$ and $75^{\text {th }}$ quartiles were 2 and 10 years respectively. About $18 \%$ of subjects were diagnosed as having DM within a year (Figure 1).

\section{Discussions}

While trying to determine cardiovascular risk among diabetic patients presenting for their first specialist visit, we observed that $41 \%$ of patients had been said to be living with DM more than 5 years prior to first specialist visit. This finding may appear disturbing considering the fact that most diabetes mellitus patients have been reported to have complications as at the time of diagnosis $[7,8]$. Specialist evaluation may be urgently needed to evaluate some of these complications. It therefore means interventions could be delayed with grave consequences. This may not necessarily be entirely true because some subjects may have been accessing health 
care at primary and secondary health care levels with satisfaction. History of hypertension was associated with DM in 51\% of all cases. We however did not ascertain if hypertension preceded DM or the other way round.

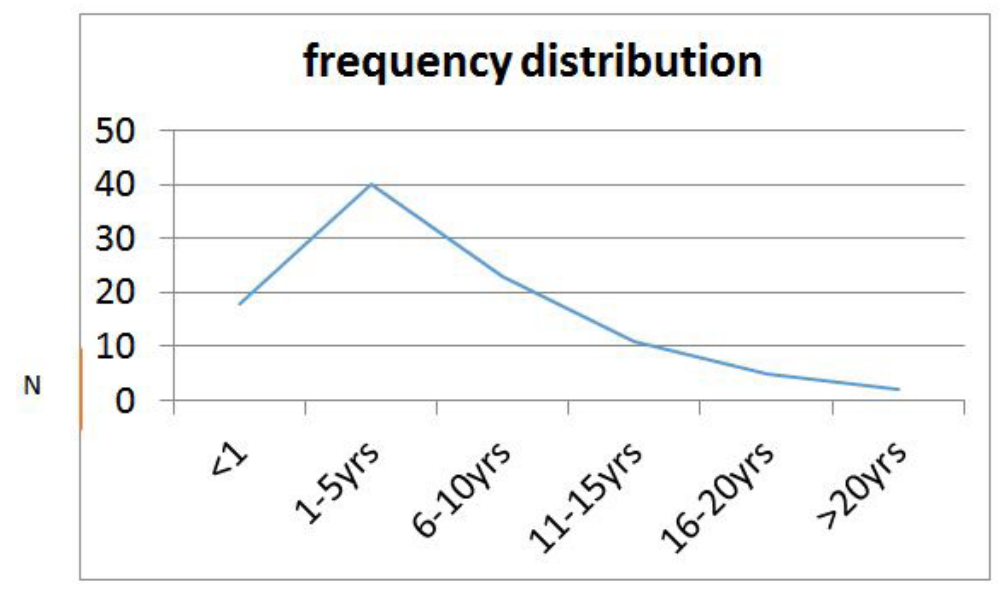

Figure 1: Means Of duration of Diabetes in the 6 Age Groups

In order to demonstrate risk association with increasing duration of illness, there was an observed relative progressive increase in means of risk factors. Age is an important factor and correlates well with duration of any chronic illness. The decline observed in waist circumference and LVM as duration gets about may not be unconnected to the fact that this subjects are likely to be elderly. Further comparing the means risk factors of each group of individuals at different stages of the disease using the analysis of variance ANOVA, shows statistical differences in the LVM, UA and creatinin. In the case of LVM, the observation may be attributed to the fact that about half the subjects were said to be hypertensive a cause of LV hypertrophy. It is also not considered as a CVD risk factor by some researchers. Uric acid and createnin on the other hand are markers of renal functions. The above finding could suggest a rapid renal and cardiac complications in asymptomatic DM subjects. Studies like that of Osunkwo in this centre had demonstrated that microangiopathy (a CVD risk) is strongly associated with duration of DM, a finding consistent with ours [13]. Danbauchi, et al. in Zaria also demonstrated left ventricular abnormality in DM patients [12,14].

This short communication was observed during further analysis of an initial study (carotid intima-media thickness study), the varying therapy these subjects might have before presenting, the skewness of duration of illness, and the relative small sample sizes are some of the limitations the could have affected the outcome of the study.

\section{Conclusion}

Most cardiovascular risks steadily rise as the duration of DM gets longer and findings suggested a higher degree of heart and renal complications as DM progresses in this environment.

\section{References}

1. Knut Borch-Johnsen (2008) Diabetes and cardiovascular disease: the challenges of prevention, diagnosis and treatment Steno Diabetes Centre, Gentofte, Denmark 20: 151-2.

2. Nathan DM, Meigs J, Singer DE (1997) The epidemiology of cardiovascular disease in type 2 diabetes mellitus: how sweet it is ... or is it? Lancet 350: 14-9.

3. Lars Rydén, Eberhard Standl, Malgorzata Bartnik, Greet Van den Berghe, John Betteridge, et al . (2007) Guidelines on diabetes, prediabetes, and cardiovascular disease. Rev Esp Cardiol 60: doi: 10.1016/S1885-5857(07)60205-9.

4. Lloyd-Jones DM, Leip EP, Larson MG, D’Agostino RB, Beiser A, et al. (2006) Prediction of lifetime risk for cardiovascular disease by risk factor burden at 50 years of age. Circulation 113: 791-8.

5. Paul Tung, Seymour R (1988) Nephropathy in NIDDM. American Journal Medicine 85: 131.

6. Remuzzi G, Schieppati A, Ruggenenti P (2002) Clinical practice. Clinical practice. Nephropathy in patients with type 2 diabetes. N Engl J Med 346: 1145-51.

7. Eckel RH, Wassef M, Chait A, Sobel B, Barrett E, et al.(2002) Prevention Conference VI: Diabetes and Cardiovascular Disease: Writing Group II: pathogenesis of atherosclerosis in diabetes. Circulation 105: e138-43.

8. Expert Committee on the Diagnosis and Classification of Diabetes Mellitus (1997) Report of the Expert Committee on the Diagnosis and Classification of Diabetes Mellitus. Diabetes Care 20: 1183-97.

9. Feller S (2010) Waist circumference gives a better prediction of diabetes risk than does BMI. Dtsch Arztebl Int 107.

10. Lorenz MW, Markus HS, Bots ML, Rosvall M, Sitzer M (2007) Prediction of clinical cardiovascular events with carotid intima-media thickness: a systematic review and meta-analysis. Circulation 115: 459-67.

11. Kitamura A, Iso H, Imano H, Ohira T, Okada T, et al. (2004) Carotid intima-media thickness and plaque characteristics as a risk factor for stroke in Japanese elderly men. Stroke 35: 2788-94.

12. Oyati AI, Danbauchi SS, Alhassan MA, Isa MS (2005) Normal values of echocardiographic parameters of apparently healthy adult Nigerians in zaria. Afr J Med Med Sci 34: 45-9.

13. Osunkwo DA, Okeahialam BN (2001) Left ventricular function in Nigerian Africans with non-insulin-dependent diabetes mellitus. Am J Cardiol 87: 1026-9. 14. Danbauchi SS, Anumah FE, Alhassan MA, David SO, Onyemelukwe GC, et al. (2005) Left ventricular function in type 2 diabetes patients without cardiac symptoms in Zaria, Nigeria. Ethn Dis 15: 635-40. 